\title{
A Collacation Method for Fredholm Integral Equations of the Second Kind
}

\author{
By E. N. Houstis and T. S. Papatheodorou
}

\begin{abstract}
An interpolation scheme based on piecewise cubic polynomials with Gaussian points as interpolation points is analyzed and applied to the solution of Fredholm equations of the second kind.
\end{abstract}

Introduction. We consider an interpolation scheme based on piecewise polynomials with continuous first derivatives and the Gaussian points as interpolation points. A collocation method based on this interpolation scheme is applied to one- and twodimensional Fredholm equations of the second kind.

This scheme has been applied to a collocation method by de Boor and Swartz [4] and Houstis [9] for the numerical solution of ordinary differential equations. Also, Douglas and Dupont [6] , [7] , [8] and Houstis [10] , [11] have studied a collocation method for partial differential equations based on the above scheme. A survey of numerical methods for the solution of Fredholm integral equations of the second kind is given by Atkinson [1].

In Part I we present the formulation and error analysis of the interpolation scheme. In Part II we apply this scheme to the solution of Fredholm integral equations of the second kind and give an experimental comparison with Nyström's method.

\section{Piecewise Cubic Hermite Interpolation at the Gaussian Points}

1. One-Dimensional Interpolation Scheme. Let $\Delta=\left(x_{i}\right)_{1}^{N+1}$ be a partition of $I \equiv[a, b], h_{i} \equiv\left|x_{i+1}-x_{i}\right|, I_{i} \equiv\left[x_{i}, x_{i+1}\right]$ and $h=\max h_{i}$. Throughout this report we denote by $P_{3}$ the set of polynomials of degree less than 4 , and $P_{3, \Delta}$ the set of functions that reduce to polynomials of degree less than 4 in each subinterval $\left[x_{i}, x_{i+1}\right]$. Also, we denote by $H_{\Delta}$ the $(2 N+2)$-dimensional vector space of all continuously differentiable piecewise cubic polynomials with respect to $\Delta$.

The Gaussian points in the subinterval $\left[x_{j}, x_{j+1}\right]$ are

$$
\xi_{2 j+i} \equiv \frac{x_{j}+x_{j+1}}{2}+\frac{(-1)^{i}}{\sqrt{3}} \frac{h_{j}}{2}, \quad i=1,2 .
$$

Let $E(I)$ be the space of real-valued functions defined on $I$. We introduce an interpolation operator $Q_{N}: E(I) \rightarrow H_{\Delta}$ such that

Received December 6, 1976; revised June 27, 1977.

AMS (MOS) subject classifications (1970). Primary 65N35.

Key words and phrases. Collocation method, integral equations. 


$$
\left(Q_{N} f\right)\left(\sigma_{l}\right)=f\left(\sigma_{l}\right), \quad l=1, \ldots, 2 N+2,
$$

where $\sigma_{1}=a, \sigma_{l}=\xi_{2 j+i}, j=1, \ldots, N, i=1,2, \sigma_{2 N+2}=b$.

This interpolation scheme is well defined. In fact, if $h(x) \in H_{\Delta}$ also interpolates $f$ as above, then $e(x) \equiv Q_{N} f(x)-h(x)$ is a cubic polynomial on $\left[x_{i}, x_{i+1}\right], 0 \leqslant i \leqslant N$, and $e\left(\sigma_{i}\right)=0,1 \leqslant i \leqslant 2 N+2$. We show that $e(x)$ is identically zero in $\left[x_{i}, x_{i+1}\right]$. If this is not so, then without loss of generality we may assume that $e(x) \neq 0$ for all $x \in\left[x_{1}, x_{2}\right]$. Rolle's Theorem implies that $e\left(x_{2}\right) D_{x} e\left(x_{2}\right)>0$. Similarly, $D_{x} e$ restricted in $\left[x_{2}, x_{3}\right]$ has roots in $\left(x_{2}, \sigma_{4}\right),\left(\sigma_{4}, \sigma_{5}\right)$. Thus, $e\left(x_{3}\right) D_{x} e\left(x_{3}\right)>0$. By induction $e\left(x_{N+1}\right) D_{x} e\left(x_{N+1}\right)>0$ contradicting the relation $e\left(x_{N+1}\right)=0$. This proves that $e(x) \equiv 0$ in $I$.

2. Two-Dimensional Interpolation Scheme. In this section we introduce a twodimensional analogue of the interpolation scheme of the previous section. Let $\Delta_{y}=$ $\left(y_{j}\right)_{1}^{M+1}$ be a partition of $[c, d], J \equiv[c, d], k_{j} \equiv\left|y_{j+1}-y_{j}\right|, J_{j} \equiv\left[y_{j}, y_{j+1}\right]$ and $k \equiv \max k_{j}$. Also, we denote by $\rho \equiv \Delta x \Delta y$ a partition of $[a, b] \times[c, d]$ and by $H_{\rho}$ the vector space of all piecewise bicubic polynomials $p(x, y)$ with respect to $\rho$, such that $D_{x}^{l} D_{y}^{\eta} p(x, y)$ is continuous on $[a, b] \times[c, d]$ for all $0 \leqslant l, \eta \leqslant 1$.

The Gaussian points in the subinterval $\left[y_{i}, y_{i+1}\right]$ are

$$
\tau_{2 i+j} \equiv \frac{y_{i}+y_{i+1}}{2}+\frac{(-1)^{j}}{\sqrt{3}} \frac{k_{i}}{2}, \quad j=1,2 .
$$

A two-dimensional interpolation operator is defined as the tensor product

$$
Q_{\rho} \equiv Q_{N} \otimes Q_{M}=Q_{N} Q_{M}
$$

3. Error Analysis. In this section, we establish a priori bounds for the interpolation scheme introduced in Section 2 for a uniform partition $\Delta$ of $[0,1]$ with mesh length $h=N^{-1}$.

Let

$$
\phi(x)= \begin{cases}(1-x)^{2}(1+2 x), & 0 \leqslant x \leqslant 1 \\ (1+x)^{2}(1-2 x), & -1 \leqslant x \leqslant 0 \\ 0, & \text { otherwise }\end{cases}
$$

and

$$
\psi(x)= \begin{cases}x(1-x)^{2}, & 0 \leqslant x \leqslant 1, \\ x(1+x)^{2}, & -1 \leqslant x \leqslant 0, \\ 0, & \text { otherwise. }\end{cases}
$$

The basis functions of the $C^{1}$ cubic piecewise polynomial space $H_{\Delta}$ are defined by

$$
\begin{aligned}
B_{2 i-1}(x)=\phi\left(\frac{x-x_{i}}{h}\right), & & 1 \leqslant i \leqslant N+1, \\
B_{2 i}(x)=h \psi\left(\frac{x-x_{i}}{h}\right), & & 1 \leqslant i \leqslant N+1 .
\end{aligned}
$$


For later use, we define the Gramian matrix

$$
G_{N} \equiv\left(B_{i}\left(\sigma_{j}\right) ; i, j=1, \ldots, 2 N+2\right)
$$

of the interpolation operator $Q_{N}$. Using the $(2 N+2) \times(2 N+2)$ matrix

$$
H_{N} \equiv\left[\begin{array}{lllllll}
1 & & & & & \\
& h & & & 0 & \\
& & \cdot & & & \\
& & & \cdot & & & \\
& 0 & & & & & \\
& & & & & \\
& & & & & h
\end{array}\right] \text {, }
$$

we find that ${ }^{\bullet}$

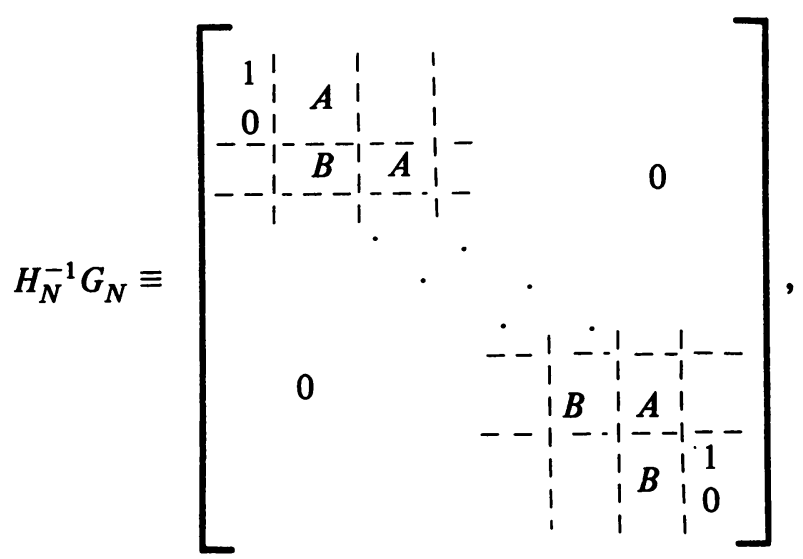

where

$$
A \equiv\left[\begin{array}{ll}
\alpha & \beta \\
\gamma & \delta
\end{array}\right], \quad B \equiv\left[\begin{array}{cc}
\beta & \alpha \\
-\delta & -\gamma
\end{array}\right]
$$

and

$$
\alpha=\frac{9+4 \sqrt{3}}{18}, \quad \beta=\frac{9-4 \sqrt{3}}{18}, \quad \gamma=\frac{3+\sqrt{3}}{36}, \quad \delta=\frac{3-\sqrt{3}}{36} .
$$

We will also use the matrix

$$
T \equiv B A^{-1}=\left[\begin{array}{cc}
-7 & 48 \\
1 & -7
\end{array}\right] \text {. }
$$

It is easy to see that for all integers $n,\left(T^{0} \equiv I\right)$,

$$
T^{n}=\left[\begin{array}{cc}
a_{n} & 48 c_{n} \\
c_{n} & a_{n}
\end{array}\right],
$$

where 
162

E. N. HOUSTIS AND T. S. PAPATHEODOROU

$$
a_{n+1}=-7 a_{n}+48 c_{n}, \quad c_{n+1}=a_{n}-7 c_{n} .
$$

More generally, from $T^{s+t}=T^{s} T^{t}$ we get

$$
\begin{array}{ll}
a_{s \pm t}=a_{s} a_{t} \pm 48 c_{s} c_{t}, & c_{s \pm t}=c_{s} a_{t} \pm a_{s} c_{t}, \\
c_{s} a_{t}=\frac{1}{2}\left(c_{s+t}+c_{s-t}\right), & c_{s} c_{t}=\frac{1}{96}\left(a_{s+t}-a_{s-t}\right), \\
a_{s} a_{t}=\frac{1}{2}\left(a_{s+t}+a_{s-t}\right), & a_{-l}=a_{l}, \quad c_{-l}=-c_{l} .
\end{array}
$$

Let $\lambda_{n} \equiv\left|a_{n}\right| c_{n} \mid=-a_{n} / c_{n}$. Since $\operatorname{det}\left(T^{n}\right)=1$, we can easily show that $\lambda_{n}$ is decreasing with $n$ and for all $n$

$$
\sqrt{48}<\lambda_{n} \leqslant 7, \quad \lambda_{1}=7
$$

$$
\begin{gathered}
c_{n}=(-1)^{n+1}\left|c_{n}\right|, \quad a_{n}=(-1)^{n}\left|a_{n}\right|, \\
\left|a_{n+1}\right|>\left|a_{n}\right|, \quad\left|c_{n+1}\right|>\left|c_{n}\right| .
\end{gathered}
$$

Since

$$
\left|a_{n}\right|=\frac{1}{2}\left(\left|c_{n+1}\right|-\left|c_{n-1}\right|\right), \quad\left|c_{n}\right|=\frac{1}{96}\left(\left|a_{n+1}\right|-\left|a_{n}\right|\right),
$$

we also have

$$
\begin{aligned}
& \sum_{l=q}^{P}\left|a_{l}\right|=\frac{1}{2}\left(\left|c_{p+1}\right|+\left|c_{p}\right|-\left|c_{q}\right|-\left|c_{q-1}\right|\right) \\
& \sum_{l=q}^{p}\left|c_{l}\right|=\frac{1}{96}\left(\left|a_{p+1}\right|+\left|a_{p}\right|-\left|a_{q}\right|-\left|a_{q-1}\right|\right) .
\end{aligned}
$$

We introduce a $(2 N+2) \times(2 N+2)$ matrix $R$ in partition form

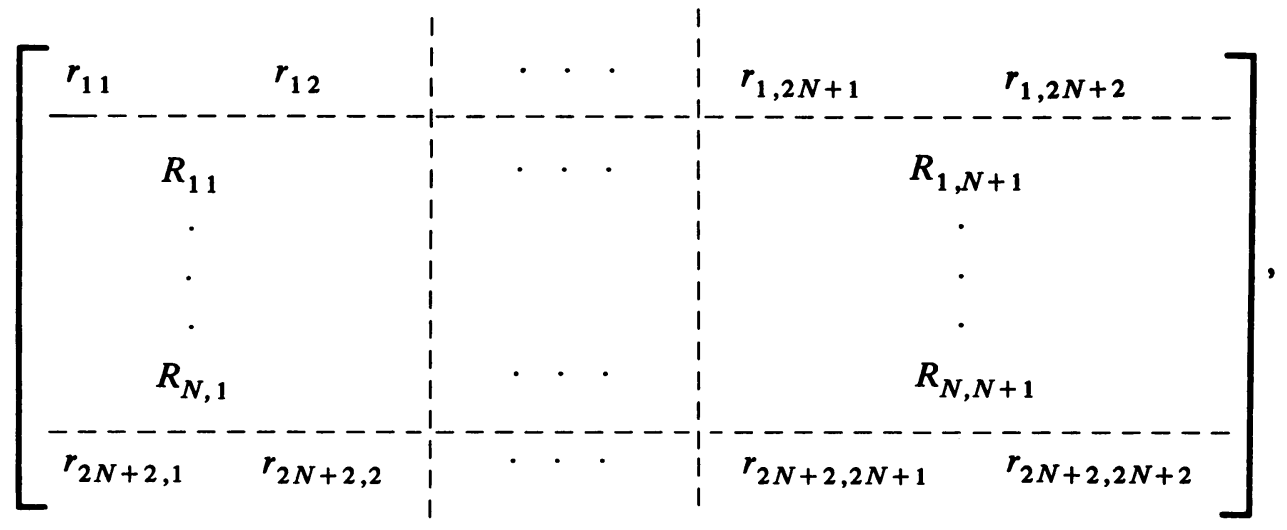


where the first and last rows are defined as

$$
\begin{aligned}
{\left[r_{1,2 j-1}, r_{1,2 j}\right] } & \equiv \frac{(-1)^{j+1}}{c_{N}}\left[c_{N-j+1} a_{N-j+1}\right], \\
{\left[r_{2 N+2,2 j-1}, r_{2 N+2,2 j}\right] } & \equiv \frac{(-1)^{N-j}}{c_{N}}\left[-c_{j-1} a_{j-1}\right],
\end{aligned}
$$

while the $2 \times 2$ matrices $R_{n, m}$ are defined as

$R_{n, m} \equiv A^{-1}\left[(-T)^{n-1} Z_{m}+\sigma_{n, m}(-T)^{n-m}\right], \quad n=1, \ldots, N, m=1, \ldots, N+1$,

with

$$
Z_{1} \equiv\left[\begin{array}{cc}
0 & \lambda_{N} \\
0 & 1
\end{array}\right], \quad Z_{m} \equiv \frac{(-1)^{m}}{c_{N}}\left[\begin{array}{cc}
c_{N-m+1} & a_{N-m+1} \\
0 & 0
\end{array}\right], \quad m=2, \ldots, N+1,
$$

and

$$
\sigma_{n, m}= \begin{cases}1 & \text { if } 2 \leqslant m \leqslant n \\ 0 & \text { otherwise }\end{cases}
$$

The reason for introducing the matrix $R$ becomes apparent by the following lemma, which shows that we have explicitly constructed the inverse of the matrix $H_{N}^{-1} G_{N}$.

Lemma 3.1. The matrix $H_{N}^{-1} G_{N}$ is invertible and its inverse is the matrix $R$.

Proof. Let $S \equiv R\left(H_{N}^{-1} G_{N}\right)$. It is enough to show that $S=I$. We partition $S$ into blocks:

$$
S=\left[\begin{array}{ccccc}
s_{11} & \tau_{11} & \cdots & \tau_{1 N} & s_{1,2 N+2} \\
\omega_{11} & s_{11} & \cdots & s_{1 N} & \omega_{1,2 N+2} \\
\cdot & \cdot & & \cdot & . \\
\cdot & \cdot & & . & \cdot \\
\cdot & \cdot & & \cdot & \cdot \\
\omega_{N 1} & S_{N 1} & & S_{N N} & \omega_{N, 2 N+2} \\
s_{2 N+2,1} & \tau_{2 N+2,1} & \cdots & \tau_{2 N+2, N} & s_{2 N+2,2 N+2}
\end{array}\right],
$$

where each $s_{i j}$ is $1 \times 1, S_{i j}$ is $2 \times 2, \omega_{i j}$ is $2 \times 1$ and $\tau_{i j}$ is $1 \times 2$. Performing the multiplication of the matrices $R$ and $H_{N}^{-1} G_{N}$, we obtain

$$
s_{11}=r_{11}=1 \text {, }
$$




$$
\begin{aligned}
\tau_{i j} & =\left[s_{1,2 j} s_{1,2 j+1}\right]=\left[r_{1,2 j-1} r_{1,2 j}\right] A+\left[r_{1,2 j-1} r_{1,2 j+2}\right] B \\
& =\frac{(-1)^{j}}{c_{N}}\left\{\left[c_{N-j+1} a_{N-j+1}\right]-\left[c_{N-j} a_{N-j}\right] T\right\} A \\
& =\frac{(-1)^{j}}{c_{N}}\left\{\left[c_{N-j+1} a_{N-j+1}\right]-\left[c_{N-j+1} a_{N-j+1}\right]\right\} A=[0,0],
\end{aligned}
$$

and

$$
s_{1,2 N+2}=r_{1,2 N+1}=0 \text {. }
$$

Similarly

$$
\omega_{i, 1}=\omega_{i, 2 N+2}=\left[\begin{array}{l}
0 \\
0
\end{array}\right], \quad \tau_{2 N+2, j}=\left[\begin{array}{ll}
0 & 0
\end{array}, \quad i, j=1, \ldots, N,\right.
$$

and

$$
s_{2 N+2,1}=0, \quad s_{2 N+2,2 N+2}=1 .
$$

For the square blocks $S_{n, m}$ we find

$$
\begin{aligned}
S_{n, m} & =R_{n, m} A+R_{n, m+1} B \\
& =A^{-1}(-T)^{n-1}\left\{Z_{m}+Z_{m+1} T+\left(\sigma_{n, m}-\sigma_{n, m+1}\right)(-T)^{1-m}\right\} A .
\end{aligned}
$$

From the definition of $Z_{m}$ and $T$ we obtain $Z_{m}+Z_{m+1} T=\delta_{1}^{m} I$. Then from the definition of $\sigma_{n, m}$ we get $S_{n, m}=\delta_{n}^{m} I$. This concludes the proof of Lemma 3.1.

LеммA 3.2. If $G_{N}$ is the Gramian of the interpolation operator $Q_{N}$, then

$$
\left\|\left(H_{N}^{-1} G_{N}\right)^{-1}\right\|_{\infty}<100
$$

for all $N \geqslant 2$.

Proof. Let

$$
\|R\|_{l} \equiv \sum_{m=1}^{2 N+2} r_{l m} \mid
$$

From the definition of $R$ and relations (3.1), (3.2), (3.3), we obtain

$$
\begin{aligned}
\|R\|_{1} & \equiv \sum_{j=1}^{N}\left(\left|r_{1,2 j+1}\right|+\left|r_{1,2 j}\right|\right) \\
& =\frac{1}{\left|c_{N}\right|} \sum_{j=1}^{N}\left(\left|c_{N-j+1}\right|+\left|a_{N-j+1}\right|\right)=\frac{1}{\left|c_{N}\right|} \sum_{l=1}^{N}\left(\left|c_{l}\right|+\left|a_{l}\right|\right) \\
& \leqslant \frac{7}{12} \frac{\left|a_{N}\right|}{\left|c_{N}\right|}+\frac{9}{2}+\frac{5}{15} \frac{\left|a_{1}\right|}{\left|c_{N}\right|}-\frac{7}{2} \frac{\left|c_{1}\right|}{\left|c_{N}\right|} \leqslant 23 / 2 .
\end{aligned}
$$

It is easy to see that $\|R\|_{2 N+2}=\|R\|_{1}$. For the remaining rows we use (3.1), (3.2) to get that for $2 \leqslant m \leqslant n$, 


$$
\begin{aligned}
A R_{n, m} & =(-T)^{n-1} Z_{m}+\sigma_{n, m}(-T)^{n-m} \\
& =\frac{1}{2\left|c_{N}\right|}\left[\begin{array}{cc}
-\left|c_{N-n-m+2}\right|+\left|c_{N-n+m}\right| & \left|a_{N-n-m+2}\right|+\left|a_{N-n+m}\right| \\
\frac{1}{48}\left(-\left|a_{N-n-m+2}\right|+\left|a_{N-n+m}\right|\right) & \left|c_{N-n-m+2}\right|+\left|c_{N-n+m}\right|
\end{array}\right],
\end{aligned}
$$

while for $n<m$

$$
A R_{n, m}=\frac{1}{2\left|c_{N}\right|}\left[\begin{array}{ll}
-\left|c_{N+n-m}\right|+\left|c_{N-n-m+2}\right| & \left|a_{N+n-m}\right|+\left|a_{N-n-m+2}\right| \\
\frac{1}{48}\left(\left|a_{N+n-m}\right|-\left|a_{N-n-m+2}\right|\right) & \left|c_{N+n-m}\right|-\left|c_{N-n-m+2}\right|
\end{array}\right]
$$

Finally, for $m=1$,

$$
A R_{n, 1}=\frac{1}{\left|c_{N}\right|}\left[\begin{array}{ll}
0 & \left|a_{N-n+1}\right| \\
0 & \left|c_{N-n+1}\right|
\end{array}\right]
$$

Using again the relations (3.1) through (3.3), we now find

$$
\begin{aligned}
& \sum_{m=1}^{N}\left\|A R_{n, m}\right\|_{1} \\
& \leqslant \frac{1}{2}\left[2 \frac{\left|a_{N-n+1}\right|}{\left|c_{N}\right|}+\frac{1}{96}\left(\frac{\left|a_{N-n+1}\right|}{c_{N} \mid}+\frac{\left|a_{N-n}\right|}{\left|c_{N}\right|}+\frac{\left|a_{N}\right|}{\left|c_{N}\right|}+\frac{\left|a_{N-1}\right|}{\left|c_{N}\right|}\right)\right. \\
& \left.+\frac{1}{2}\left(\frac{\left|c_{N-n}\right|}{\left|c_{N}\right|}+9+\frac{\left|c_{N-1}\right|}{\left\|c_{N}\right\|}+\frac{\left|a_{N}\right|}{\left|c_{N}\right|}\right)\right] \leqslant \frac{35}{3}
\end{aligned}
$$

and

$$
\begin{aligned}
\sum_{m=1}^{N}\left\|A R_{n, m}\right\|_{2} \leqslant \frac{1}{2}\left[2 \frac{\left|c_{N-n+1}\right|}{\left|c_{N}\right|}+\frac{1}{96}\left(\frac{\left|a_{N-n}\right|}{\left|c_{N}\right|}+9 \frac{\left|a_{N}\right|}{\left|c_{N}\right|}+48+\frac{\left|c_{N-n+1}\right|}{\left|c_{N}\right|}\right.\right. \\
\left.\left.+\frac{\left|c_{N-n}\right|}{\left|c_{N}\right|}+\frac{\left|a_{N-1}\right|}{\left|c_{N}\right|}+\frac{\left|a_{N}\right|}{\left|c_{N}\right|}+\frac{\left|c_{N-1}\right|}{\left|c_{N}\right|}\right)\right] \leqslant 2 .
\end{aligned}
$$

By definition now, we have for $l=1,2$,

$$
\|R\|_{2 n+l}=\sum_{m=1}^{N}\left\|A^{-1} A R_{n, m}\right\|_{l} \leqslant \sum_{m=1}^{N}\left\|A^{-1}\right\|_{\infty}\left\|A R_{n, m}\right\|_{l},
$$


while $\left\|A^{-1}\right\|_{\infty}=(7 \sqrt{3}+9) / 4$. Thus, for the norm $\|R\|_{\infty}=\max _{i}\|R\|_{i}$ the following bound holds

$$
\|R\|_{\infty}=\left\|\left(H^{-1} G_{N}\right)^{-1}\right\|_{\infty}<100
$$

This concludes the proof of Lemma 3.2.

Remark. As the proof of Lemma 3.2 suggests, the bound (3.4) can be improved. Our conjecture is that a more careful analysis will show that the norm $\left\|\left(H_{N}^{-1} G_{N}\right)^{-1}\right\|_{\infty}$ is decreasing in $N$, that

$$
\lim _{N \rightarrow \infty}\left\|\left(H_{N}^{-1} G_{N}\right)^{-1}\right\|_{\infty}=\frac{69-29 \sqrt{3}}{2}
$$

and that for all $N \geqslant 2$

$$
\frac{69-29 \sqrt{3}}{2} \leqslant\left\|\left(H_{N}^{-1} G_{N}\right)^{-1}\right\|_{\infty} \leqslant\left\|H_{2}^{-1} G_{2}\right\|_{\infty}=\frac{33 \sqrt{3}+9}{7} .
$$

Numerical experiments confirm this conjecture.

LeмmA 3.3. Let $Q_{N}$ be the interpolation operator defined by (1.2). Then $Q_{N}$ is bounded in the $L_{\infty}$-norm.

Proof. We can express the $Q_{N} f$ interpolant as

$$
\left(Q_{N} f\right)(x)=\sum_{i=1}^{N+1}\left\{a_{i} B_{2 i-1}(x)+b_{i} B_{2 i}(x) / h\right\}
$$

where the $B_{i}$ 's are the previously defined basis functions of $H_{\Delta}$. Let $f$ denote the column vector $\left\{f\left(\sigma_{j}\right)\right\}_{j=1}^{2 N+2}$ and set $L_{N}=\left(H_{N}^{-1} G_{N}\right)^{T}$. Then,

$$
\mathbf{f}=L_{N}\left[\begin{array}{c}
a_{1} \\
b_{1} \\
a_{2} \\
b_{2} \\
a_{3} \\
b_{3} \\
\cdot \\
\cdot \\
\cdot
\end{array}\right]
$$

In Lemma 3.2 we prove that $\left\|L_{N}^{-1}\right\|_{\infty}<100$; therefore,

$$
\max _{i}\left(\max \left(\left|a_{i}\right|,\left|b_{i}\right|\right)\right) \leqslant 100\|f\|_{L_{\infty}}
$$

For all $x \in\left[x_{i}, x_{i+1}\right], 1 \leqslant i \leqslant N$, we have

$$
\left(Q_{N} f\right)(x)=a_{i} B_{2 i-1}(x)+b_{i} B_{2 i}(x) / h+a_{i+1} B_{2 i+1}(x)+b_{i+1} B_{2 i+2}(x) / h ;
$$

and since $B_{2 i-1}, B_{2 i} / h$ are bounded by unity, we obtain 


$$
\left\|Q_{N} f\right\|_{L_{\infty}} \leqslant 400\|f\|_{L_{\infty}}
$$

THEOREM 3.1. If $f \in W^{s, \infty}(I), s=0,1,2,3$, or 4 , then

(i) $Q_{N} f \rightarrow f$, as $N \rightarrow \infty$, and

(ii) for the interpolation error we have

$$
\left\|Q_{N} f-f\right\|_{L_{\infty}} \leqslant c h^{s}\left\|D^{s} f\right\|_{L_{\infty}},
$$

where $c$ is independent of $h$.

Proof. Let $\partial_{H} f$ be the Hermite interpolant of $f$, defined by interpolation of $f$ and its first derivative at the nodes of the partition $\Delta$. From the triangle inequality we find

$$
\left\|f-Q_{N} f\right\|_{L_{\infty}} \leqslant\left(1+\left\|Q_{N}\right\|\right)\left\|f-\partial_{H} f\right\|_{L_{\infty}} .
$$

Moreover, for the Hermite interpolation error, it is known [3, p. 236]

$$
\left\|f-\partial_{H} f\right\|_{L_{\infty}} \leqslant c h^{s}\left\|D^{s} f\right\|_{L_{\infty}}, \quad s>0 .
$$

From (3.5), (3.6), (3.7) and Lemma 3.3, we now get

$$
\left\|f-Q_{N} f\right\|_{L_{\infty}} \leqslant c h^{s}\left\|D^{s} f\right\|_{L_{\infty}}
$$

This proves conclusions (i) and (ii).

THEOREM 3.2. If $f \in W^{s, \infty}(\Omega)$, $s=1,2,3,4$, and $\Omega=[0,1]^{2}$, then

$$
\left\|f-Q_{\rho} f\right\|_{L_{\infty}} \leqslant c\left(h^{s}\left\|D_{x}^{s} f\right\|_{L_{\infty}}+h^{p} k^{q}\left\|D_{x}^{p} D_{y}^{q} f\right\|_{L_{\infty}}+k^{s}\left\|D_{y}^{s} f\right\|_{L_{\infty}}\right)
$$

$$
\leqslant c \rho^{s}\|f\|_{w^{s, \infty}}, \quad \text { where } s=p+q
$$

and $p, q$ are integers.

Proof. From the triangle inequality we have

$$
\begin{aligned}
\left\|f-Q_{\rho} f\right\|_{L_{\infty}} & \leqslant\left\|f-Q_{N} f\right\|_{L_{\infty}}+\left\|Q_{N}\left(f-Q_{M} f\right)\right\|_{L_{\infty}} \\
& \leqslant\left\|f-Q_{N} f\right\|_{L_{\infty}}+\left\|Q_{N}\left(f-Q_{M} f\right)-\left(f-Q_{M} f\right)\right\|_{L_{\infty}}+\left\|f-Q_{M} f\right\|_{L_{\infty}} .
\end{aligned}
$$

Using the results of Theorem 3.1, we now get

$$
\begin{aligned}
\left\|f-Q_{\rho} f\right\|_{L_{\infty}} & \leqslant h^{s}\left\|D_{x}^{s} f\right\|_{L_{\infty}}+h^{p}\left\|D_{x}^{p}\left(f-Q_{M} f\right)\right\|_{L_{\infty}}+k^{s}\left\|D_{y}^{s} f\right\|_{L_{\infty}} \\
& \leqslant h^{s}\left\|D_{x}^{s} f\right\|_{L_{\infty}}+h^{p} k^{q}\left\|D_{x}^{p} D_{y}^{q} f\right\|_{L_{\infty}}+k^{s}\left\|D_{y}^{s} f\right\|_{L_{\infty}} \\
& \leqslant c \rho^{s}\|f\|_{w^{s, \infty}}
\end{aligned}
$$

which completes the proof of the theorem. 


\section{Two-Dimensional Collocation}

4. Procedure and Error Estimation. In this section we consider the problem of approximating the solution of the integral equation

$$
\Lambda u=u(P)-\lambda \int_{\Omega} k(P ; Q) u(Q) d Q=f(P) \quad \text { where } \Omega=[0,1]^{2}
$$

and, for brevity, $P=(x, y), Q=(s, t)$ and $d Q=d s d t$.

Let

$$
K u \equiv \int_{\Omega} k(P ; Q) u(Q) d Q
$$

We seek an approximation $u_{\rho} \in H_{\rho}$ to $u$ of the form

$$
u_{\rho}(P) \equiv \sum_{i=1}^{2 N+2} \sum_{j=1}^{2 M+2} \alpha_{i j} B_{i}(x) B_{j}(y)
$$

such that

$$
\left(I-\lambda Q_{\rho} K\right) u_{\rho}=Q_{\rho} f
$$

THEOREM 4.1. If

A1. $\lambda$ is not an eigenvalue of the kernel $k(P ; Q)$.

A2. The right side and the kernel of Eq. (4.1) are in $W^{s, \infty}(\Omega), s=1,2,3$, or 4 , then

(i) for sufficiently small $|\rho|$ the collocation system (4.3) is uniquely solvable and

(ii) for the error of approximation we have

$$
\left\|u-u_{\rho}\right\|_{L_{\infty}} \leqslant c\left\|u-Q_{\rho} u\right\|_{L_{\infty}} \leqslant c \rho^{s}\|u\|_{W^{s, \infty}} .
$$

Proof. By the definition of the operator $K$ we obtain

$$
\left\|K u-Q_{\rho} K u\right\|_{L_{\infty}}=\left\|\int_{\Omega}\left(k(\cdot, Q)-Q_{\rho} k(\cdot, Q)\right) u d Q\right\|_{L_{\infty}}
$$

and since $\left\|k(\cdot Q)-Q_{\rho} k(\cdot, Q)\right\|_{L_{\infty}} \rightarrow 0$ as $|\rho| \longrightarrow 0$ it follows from (4.5) that

$$
\left\|K-Q_{\rho} K\right\| \rightarrow 0 \text { as }|\rho| \rightarrow 0 .
$$

In fact, for any $\epsilon>0$ there exists $\delta_{0}(\epsilon)$ such that for $|\rho|<\delta_{0}(\epsilon)$ the inequality $\left\|K-Q_{\rho} K\right\|<\epsilon$ holds. But in this case, on the sphere $\|u\|_{L_{\infty}}=1$ we have

$$
\left\|\left(I-\lambda Q_{\rho} K\right) u\right\|_{L_{\infty}} \geqslant\|(I-\lambda K) u\|_{L_{\infty}}-|\lambda|\left\|\left(K-Q_{\rho} K\right) u\right\|_{L_{\infty}} \geqslant \alpha-|\lambda| \epsilon,
$$

where $0<\alpha \leqslant \inf _{\|u\|_{L_{\infty}}=1}\|(I-\lambda K) u\|_{L_{\infty}}$.

Consequently, for sufficiently small $|\rho|$, the relationship

$$
\inf _{\|u\|_{L_{\infty}}=1}\left\|\left(I-\lambda Q_{\rho} K\right) u\right\|_{L_{\infty}} \geqslant \beta>0
$$

holds, from which conclusion (i) follows. 
Now from (4.3) it follows easily that

$$
u-u_{\rho}=\left(I-\lambda Q_{\rho}\right)^{-1}\left(u-Q_{\rho} u\right)
$$

from which

$$
\left\|u-u_{\rho}\right\|_{L_{\infty}} \leqslant\left\|\left(I-\lambda Q_{\rho} K\right)^{-1}\right\|\left\|u-Q_{\rho} u\right\|_{L_{\infty}} \leqslant \frac{1}{\beta}\left\|u-Q_{\rho} u\right\|_{L_{\infty}}
$$

and combining this inequality with that of (3.8) we obtain (4.4). This concludes the proof of Theorem 4.1 .

Finally, we remark that the above results also hold for one-dimensional integral equations of the second kind.

5. Numerical Results. In this section we present some numerical results concerning the approximation of the solution of some one-dimensional integral equations taken from [2]. The numerical solutions are computed by one-dimensional analogue of the collocation scheme introduced in Part II with a three-point Gaussian rule and by Nyström's method with Simpson's numerical integration rule [1]. The partition $\Delta$ used is uniform with mesh size $h=1 / N$. The rate of convergence estimate

$$
\log \left(\frac{\text { error for } h}{\text { error for } h / 2}\right) / \log 2
$$

is also given.

The integral equation

$$
u(s)-\lambda \int_{a}^{b} k(s, t) u(t) d t=f(s), \quad a \leqslant s \leqslant b,
$$

is solved for various kernel functions $k$, right side functions $f$ and parameters $\lambda$.

Case (i). $k(s, t)=\cos (\pi s t), 0<s, t \leqslant 1, \lambda=1$. The right side $f$ is chosen so that $u(s)=e^{s} \cos (\pi s)$.

\begin{tabular}{|c|l|l|l|l|}
\hline & \multicolumn{2}{|c|}{ COLLOCATION } & \multicolumn{2}{c|}{ NYSTROM } \\
\hline$N$ & Max. Error & Rate & Max. Error & Rate \\
\hline 3 & $4.55 \times 10^{-2}$ & & $1.18 \times 10^{-2}$ & \\
\hline 6 & $4.24 \times 10^{-3}$ & 3.4 & $5.85 \times 10^{-4}$ & 4.3 \\
\hline 12 & $3.37 \times 10^{-4}$ & 3.7 & $3.47 \times 10^{-5}$ & 4.1 \\
\hline 24 & $2.59 \times 10^{-5}$ & 3.7 & $2.14 \times 10^{-6}$ & 4. \\
\hline 48 & $1.75 \times 10^{-6}$ & 3.9 & $1.33 \times 10^{-7}$ & 4. \\
\hline
\end{tabular}

Case (ii). $k(s, t)=e^{\beta s t}, 0 \leqslant s, t \leqslant 1, \lambda=1$. For the numerical example we pick $f$ so that

$$
u(s)=e^{\alpha s}, \quad \alpha=1, \beta=5
$$




\begin{tabular}{|c|l|l|l|l|}
\hline & \multicolumn{2}{|c|}{ COLIOCATION } & \multicolumn{2}{c|}{ NYSTROM } \\
\hline $\mathrm{N}$ & Max. Error & Rate & Max. Error & Rate \\
\hline 3 & $1.33 \times 10^{-3}$ & & $1.60 \times 10^{-1}$ & \\
\hline 6 & $2.17 \times 10^{-5}$ & 5.9 & $1.55^{\times 10^{-2}}$ & 3.37 \\
\hline 12 & $2.72 \times 10^{-7}$ & 6.3 & $1.02 \times 10^{-3}$ & 3.90 \\
\hline 24 & $1.1 \times 10^{-8}$ & 4.6 & $6.49 \times 10^{-5}$ & 3.97 \\
\hline 48 & $8.57 \times 10^{-10}$ & 3.7 & $4.06 \times 10^{-6}$ & 4.00 \\
\hline
\end{tabular}

Case (iii). $k(s, t)=t-s, 0 \leqslant t, s \leqslant 1, \lambda=1$. Choose $f$ so that

$$
u(s)=s^{\alpha / 2}, \quad \alpha=1,3,5,7,9
$$

\begin{tabular}{|c|c|c||l|l|}
\hline$\alpha=1$ & \multicolumn{2}{|c|}{ COLLOCATION } & \multicolumn{2}{c|}{ NYSTROM } \\
\hline $\mathrm{N}$ & Max. Error & Rate & Max. Frror & Rate \\
\hline 3 & $9.79 \times 10^{-3}$ & .5 & $4.32 \times 10^{-3}$ & \\
\hline 6 & $6.92 \times 10^{-3}$ & .5 & $1.52 \times 10^{-3}$ & 1.5 \\
\hline 12 & $4.87 \times 10^{-3}$ & .5 & $5.33 \times 10^{-4}$ & 1.5 \\
\hline 24 & $3.43 \times 10^{-3}$ & .5 & $1.88 \times 10^{-4}$ & 1.5 \\
\hline 48 & $2.42 \times 10^{-3}$ & .5 & $6.66 \times 10^{-5}$ & 1.5 \\
\hline
\end{tabular}

\begin{tabular}{|c|l|l|l|l|}
\hline$\alpha=3$ & COLLOCATION & \multicolumn{2}{|c|}{ NYSTROM } \\
\hline$N$ & Max. Error & Rate & Max. Error & Rate \\
\hline 3 & $4.21 \times 10^{-4}$ & & $1.36 \times 10^{-4}$ & \\
\hline 6 & $1.46 \times 10^{-4}$ & 1.5 & $2.30 \times 10^{-5}$ & 2.60 \\
\hline 12 & $5.10 \times 10^{-5}$ & 1.5 & $3.95 \times 10^{-6}$ & 2.54 \\
\hline 24 & $1.79 \times 10^{-5}$ & 1.5 & $6.90 \times 10^{-7}$ & 2.52 \\
\hline 48 & $6.32 \times 10^{-6}$ & 1.5 & $1.17 \times 10^{-7}$ & 2.56 \\
\hline
\end{tabular}


FREDHOLM INTEGRAL EQUATIONS OF THE SECOND KIND

\begin{tabular}{|c|l|c||c|c|}
\hline$\alpha=5$ & \multicolumn{2}{|l||}{ COLLCATION } & \multicolumn{2}{|c|}{ NYSTROM } \\
\hline$N$ & Max. Error & Rate & Max. Error & Rate \\
\hline 3. & $1.05 \times 10^{-4}$ & & $1.03 \times 10^{-3}$ & \\
\hline 6 & $1.82 \times 10^{-5}$ & 2.5 & $7.55 \times 10^{-5}$ & 3.77 \\
\hline 12 & $3.21 \times 10^{-6}$ & 2.5 & $5.25 \times 10^{-6}$ & 3.85 \\
\hline 24 & $5.64 \times 10^{-7}$ & 2.5 & $3.66 \times 10^{-7}$ & 3.84 \\
\hline 48 & $9.95 \times 10^{-8}$ & 2.5 & $2.57 \times 10^{-8}$ & 3.83 \\
\hline
\end{tabular}

\begin{tabular}{|l|l|l|l|l|}
\hline$\alpha=7$ & COLLOCATION & \multicolumn{2}{|c|}{ NYSTROM } \\
\hline$N$ & Max. Error & Rate & Max. Error & Rate \\
\hline 3 & $1.61 \times 10^{-4}$ & & $2.22 \times 10^{-4}$ & \\
\hline 6 & $1.50 \times 10^{-4}$ & 3.5 & $1.37 \times 10^{-5}$ & 4. \\
\hline 12 & $1.24 \times 10^{-6}$ & 3.5 & $8.52 \times 10^{-7}$ & 4. \\
\hline 24 & $1.09 \times 10^{-7}$ & 3.5 & $5.31 \times 10^{-8}$ & 4. \\
\hline 48 & $9.74 \times 10^{-9}$ & 3.5 & $3.3 \times 10^{-9}$ & 4. \\
\hline
\end{tabular}

\begin{tabular}{|c||c|c||l|l|}
\hline$\alpha=9$ & \multicolumn{2}{|c|}{ COLLOCATION } & \multicolumn{2}{|c|}{ NYSTROM } \\
\hline $\mathrm{N}$ & Max. Error & Rate & Max. Error & Rate \\
\hline 3 & $4.32 \times 10^{-4}$ & & $4.56 \times 10^{-4}$ & \\
\hline 6 & $4.66 \times 10^{-5}$ & 4.64 & $2.88 \times 10^{-5}$ & 3.98 \\
\hline 12 & $6.97 \times 10^{-7}$ & 4.74 & $1.80 \times 10^{-6}$ & 4. \\
\hline 24 & $2.61 \times 10^{-8}$ & 4.74 & $1.12 \times 10^{-7}$ & 4. \\
\hline 48 & $8.75 \times 10^{70}$ & 4.90 & $7.00: 10^{-9}$ & 4. \\
\hline
\end{tabular}

Case (iv).

$$
\lambda=.3 \text { and } k(s, t)= \begin{cases}-s(1-t), & 0 \leqslant s \leqslant t<1 \\ -t(1-s), & 0 \leqslant t \leqslant s<1 .\end{cases}
$$


Choose $f$ so that

$$
u(s)=25 s^{5}(1-s)
$$

\begin{tabular}{|l|l|l|l|l|}
\hline & \multicolumn{2}{|l|}{ COLLOCATION } & \multicolumn{2}{|c|}{ NYSTROM } \\
\hline$N$ & Max. Error & Rate & Max. Error & Rate \\
\hline 3 & $4.75 \times 10^{-2}$ & & $3.39 \times 10^{-3}$ & \\
\hline 6 & $4.05 \times 10^{-3}$ & 3.6 & $5.69 \times 10^{-4}$ & 2.6 \\
\hline 12 & $3.09 \times 10^{-4}$ & 3.7 & $1.44 \times 10^{-4}$ & 2. \\
\hline 24 & $2.49 \times 10^{-5}$ & 3.6 & $3.61 \times 10^{-5}$ & 2. \\
\hline 48 & $2.22 \times 10^{-6}$ & 3.5 & $9.01 \times 10^{-6}$ & 2. \\
\hline
\end{tabular}

Case (v). $k(s, t)=t-s, 0 \leqslant s, t \leqslant 1, \lambda=1$.

Choose $f$ so that

$$
u(s)=s \ln (s)
$$

\begin{tabular}{|l|ll|l|l|l|}
\hline \multicolumn{3}{|l|}{ COLLOCATION } & \multicolumn{3}{|c|}{ NYSTROM } \\
\hline N & Max. Error & Rate & Max. Error & Rate \\
\hline 3 & 1.45 & $10^{-2}$ & & $1.7310^{-3}$ & \\
\hline 6 & 7.24 & $10^{-3}$ & 1. & $4.2210^{-4}$ & 2. \\
\hline 12 & 3.63 & $10^{-3}$ & 1. & $1.0410^{-4}$ & 2. \\
\hline 24 & 1.86 & $10^{-3}$ & 1. & $2.5010^{-5}$ & 2. \\
\hline 48 & 9.09 & $10^{-4}$ & 1. & $6.4510^{-6}$ & 1.95 \\
\hline
\end{tabular}

The estimated rates of convergence in Cases (i)-(v) are in good agreement with those suggested by Theorem 4.1 depending on the smoothness of the solution.

The above data indicate that the collocation method is faster than Nyström for problems with smooth solutions and nonsmooth kernels. The Nyström method runs faster in the cases of nonsmooth solutions but we believe that collocation with nonuniform mesh will do equally well. It is worth noting that for both methods all the time is spent in solving the linear system.

The Cases (i)-(v) were solved by the collocation method described in this paper with a two-point Gaussian rule. The results obtained are less accurate, but the computed rate estimates agree with the a priori estimate obtained in Theorem 4.1 as expected. 
All the numerical experiments were carried out on a CDC 6500 in single precision.

Department of Computer Sciences Purdue University

West Lafayette, Indiana 47907

Department of Mathematics Clarkson College of Technology Potsdam, New York 13676

1. K. E. ATKINSON, A Survey of Numerical Methods for the Solution of Fredholm Integral Equations of the Second Kind, Dept. of Math., Univ. of Iowa publication.

2. K. E. ATKINSON, "An automatic program for linear Fredholm integral equations of the second kind," ACM Trans. Math. Software, v. 2, 1976, pp. 154-171.

3. G. BIRKHOFF, M. H. SCHULTZ \& R. S. VARGA, "Piecewise Hermite interpolation in one and two variables with applications to partial differential equations," Numer. Math., v. 11, 1968 , pp. $232-256$.

4. C. de BOOR \& B. SWARTZ, "Collocation at Gaussian points," SIAM J. Numer. Anal., v. 10,1973 , pp. $582-606$.

5. L. M. DELVES \& J. WALSH, Numerical Solution of Integral Equations, Clarendon Press, Oxford, 1974.

6. JIM DOUGLAS, JR. \& TODD DUPONT, "A finite element collocation method for quasilinear parabolic equations," Math. Comp., v. 27, 1973, pp. 17-28.

7. JIM DOUGLAS, JR. \& TODD DUPONT, "A super convergence result for the approximate solution of the heat equation by a collocation method," The Mathematical Foundations of the Finite Element Method with Applications to Partial Differential Equations (A. K. Aziz, Editor), Academic Press, New York, 1972.

8. JIM DOUGLAS, JR. \& TODD DUPONT, Collocation Methods for Parabolic Equations in a Single Space Variable (based on $C^{1}$-Piecewise-Polynomial Spaces), Lecture Notes in Math., vol. 385, Springer-Verlag, Berlin and New York, 1974.

9. E. N. HOUSTIS, "A collocation method for systems of nonlinear ordinary differential equations," J. Math. Anal. Appl., v. 61, 1978.

10. E. N. HOUSTIS, "Application of method of collocation on lines for solving nonlinear hyperbolic problems," Math. Comp., v. 31, 1977, pp. 443-456. (To appear.)

11. E. N. HOUSTIS, "Collocation methods for linear elliptic problems," Math. Comp.

12. E. N. HOUSTIS \& T. S. PAPATHEODOROU, Piecewise Cubic Hermite Interpolation at the Gaussian Points, Purdue University, CSD-TR 199, July 1976.

13. M. H. SCHULTZ, Spline Analysis, Prentice-Hall, Englewood Cliffs, N. J., 1973. 